\title{
Lower urinary tract symptoms associated with neurological conditions: Observations on a clinical sample of outpatients neurorehabilitation service
}

\author{
Fabrizio Torelli ${ }^{1}$, Erica Terragni ${ }^{2}$, Salvatore Blanco ${ }^{1}$, Natale Di Bella ${ }^{2}$, Marco Grasso ${ }^{1}$, Donatella Bonaiuti ${ }^{2}$ \\ ${ }^{1}$ Division of Urology and. ${ }^{2}$ Division of Neurorehabilitation, San Gerardo Hospital, Monza, Italy.
}

\begin{abstract}
Summary Objectives: The overall aims of this study were to investigate the lower urinary tract symptoms (LUTS) associated with neurological conditions and their prevalence and impact on a clinical sample of outpatients of a neurorehabilitation service.

Materials and methods: We reviewed the files of 132 patients treated in our neurorehabilitation service from December 2012 to December 2013. Patients were divided into several subgroups based on the neurological diagnosis: Multiple Sclerosis (MS), other demyelinating diseases, Peripheral Neuropathy, neurovascular disorders (ND), neoplastic disease, traumatic brain injury (TBI), Parkinson and Parkinsonism, spinal cord injuries (SCI). Urinary status was based on medical evaluations of history of LUTS, type, degree, onset and duration of symptoms. We tried to analyze prevalence, kind of disorder, timing of presentation (if before or after the neurological onset) and eventual persistence of urological disorders (in the main group and in all subgroups). Results: At the time of admission to our rehabilitation service, LUTS were observed in 14 out of 132 cases (11\%). A high proportion of these outpatients (64.2\%) presented bothersome urinary symptoms such as incontinence, frequency and urgency (storage LUTS). The most frequent symptom was urinary urge incontinence $(42.8 \%)$. This symptom was found to be prevalent in the multiple sclerosis and neurovascular disorders. In 93\% the urinary symptoms arose as a result of neurologic conditions and $78.5 \%$ did not present a complete recovery of urological symptoms in spite of improved selfreported functional activity limitations. None of these patients performed urological rehabilitation.

Conclusions: Neurological disorders are a significant issue in rehabilitation services and it can lead to lower tract dysfunction, which causes LUTS. Storage symptoms are more common, especially urge incontinence. Current literature reports that a further optimization of the rehabilitation potential of neurologically ill patients is possible through an implementation of urological basic measures into the neurological treatment routine.
\end{abstract}

KEY WORDS: Neurorehabilitation service; Outpatient; Neurovascular disorder; Lower urinary tract symptoms (LUTS); Lower urinary tract dysfunction (LUTD); Urge incontinence.

Submitted 17 November 2014; Accepted 28 January 2015

\section{INTRODUCTION}

In the practice of physical medicine and rehabilitation, neurologic conditions, such as cerebrovascular accident
(CVA), neurodegenerative disease due to Parkinson's disease (PD), multiple sclerosis (MS),spinal cord injury (SCI), traumatic brain injury (TBI) or others are frequently associated with Lower urinary tract dysfunction (LUTD). It is a well-known fact that many different neurologic disorders can cause LUTD through the development of lesions in different nerve centres. Lower urinary tract symptoms (LUTS) can include storage, voiding, and post-micturition symptoms (1). Although incomplete emptying/urinary retention can occur, generally storage disorders are the most common urinary symptoms (2). Frequency, urgency and urinary incontinence can cause social embarrassment, reduce quality of life (QoL) and may also cause difficulties in rehabilitative procedures (3). Patients suffering from urological symptoms without adequate treatment may significantly lose quality of life, both at medical and at subjective assessment and to be qualified as a complex entity in terms of rehabilitation training and economical care (4).

The purposes of this study were to describe the characteristics of the urological disorders associated with neurological conditions treated in outpatient setting in a service of neurorehabilitation and to identify the prevalence of specific urological disorders in the group, in order to improve cooperation between the medical specialist in rehabilitation and urologists.

\section{MATERIALS AND METHODS}

We reviewed the files of 132 patients treated in our neurorehabilitation service from December 2012 to December 2013. Details characteristics of the sample are outlined in Table 1. Patients were divided into several subgroups based on the neurological diagnosis: Multiple Sclerosis (MS), other demyelinating diseases, Peripheral Neuropathy, neurovascular disorders (ND), neoplastic disease, traumatic brain injury (TBI), Parkinson and Parkinsonism, spinal cord injuries (SCI). Urinary status was based on medical evaluations of history of LUTS, type, degree, onset and duration of symptoms. In general, symptoms and concurrent medical conditions are described during the patient interview. LUTS are defined following the International Continence Society standard terminology and so divided into three groups: storage, 
voiding, and post micturition symptoms (5). Functional status refers to an individual capability or level of skill in performing basic tasks to daily living. Data on motor (self-care, sphincter control, locomotion and mobility) and cognitive domain (communication and social cognition scale) were collected to compare concurrent urological conditions. Large cognitive impairment and functional disability were not included. We tried to analyze prevalence, kind of disorder, timing of presentation (if before or after the neurological onset) and eventual persistence of urological disorders (in the main group and in all subgroups). All the patients in this study using bladder catheter were classified as having urinary voiding symptoms. Among the analyzed disorders, we excluded urinary tract infections (UTI), because we considered those as a confounding factor.

Table 1.

Characteristics of the sample (Neurorehabilitation service, San Gerardo Hospital Monza-Italy).

\begin{tabular}{|lc|}
\hline Characteristics & Sample (132 pts December 2012-2013) \\
\hline Socio-demographics & $26-81$ \\
Range age & 68 \\
Mean age & 86 \\
\hline Gender & 46 \\
Females & \\
Males & \\
\hline
\end{tabular}

Table 2.

Details of storage and voiding urinary symptoms.

\begin{tabular}{|lcc|}
\hline Clinically urinary symptoms & $\begin{array}{c}\text { Neurologic population (14 pts) } \\
\text { N" }\end{array}$ \\
\hline Urinary urge incontinence & 6 & 42.8 \\
\hline Increased daytime frequency & 2 & 14.3 \\
\hline Urgency, frequency, straining, slow stream & 1 & 7.1 \\
\hline Straining, hesitancy, slow stream & 4 & 28.5 \\
\hline $\begin{array}{l}\text { Feeling of incomplete emptying, } \\
\text { post micturition dribble }\end{array}$ & 1 & 7.1 \\
\hline
\end{tabular}

\section{RESULTS}

At the time of admission to our rehabilitation service, LUTS were observed in 14 out of 132 cases (11\%); 118 patients (89\%) did not show relevant urological symptoms. Among the 14 patients with urological symptoms, 8 presented storage symptoms, while 5 were affected by voiding and post-micturition symptoms. One patient showed both storage and voiding urinary symptoms. Among those affected by voiding urinary symptoms, only one exhibited indwelling urinary catheter by hospital discharge. Specific urinary symptoms are listed in Table 2. In 13 out of 14 patients, the urinary disease was related to the acute neurological onset, while in one patient the problem was pre-existing. In 11 out of 14 patients the urinary disorders currently persist, in 3 out of them the urinary problem was solved. Among the 14 affected patients, 6 presented MS, 7 ND and 1 SCI.

Among all patients included in this study, 18 had been diagnosed MS (13.6\%). Between them 6 patients (33.3\%) presented storage urinary symptoms, of which 3 (16.6\% of the total) with incontinence, 2 (11.1\%) with only urgency and increased daytime urinary frequency and 1 (5.5\%) with both storage and voiding urinary symptoms. In all patients LUTS currently persists. Seventy-one out of $132(53,8 \%)$ patients were treated in our service for the rehabilitation of cerebrovascular diseases. Seven of them (10\%) presented urinary disorders, of which $3(42.8 \%)$ storage urinary symptoms (all of them suffer from incontinence as well). Four of them (57\%) voiding urinary symptoms (3 with straining, hesitancy and slow stream and another maintained bladder catheter for urinary retention at hospital discharge). Only one patient presented the problem before the acute neurological event. Among the 7 patients affected, in 3 the problem was solved, whereas in 4 the problem currently persists.

Another case of urinary disorder presented spinal cord injury. Although this neurogenic bladder patient performed the combination treatment of the Credé technique, tapping, and /or Valsalva maneuver, the feeling of incomplete emptying, post micturition dribble are currently persisting. However, data on the prevalence and relationship of urinary symptoms with neurological conditions experienced in our retrospective study are further reported in Table 3.

Table 3.

Prevalence and relationship of urinary symptoms among outpatients neurorehabilitation service compared with neurologic conditions.

\begin{tabular}{|c|c|c|c|c|c|c|c|c|c|}
\hline \multirow[t]{2}{*}{ Diagnosis } & \multirow[t]{2}{*}{$\%$ of total diagnosis } & \multirow[t]{2}{*}{ Number affected } & \multicolumn{3}{|c|}{ Type of urinary disorder } & \multicolumn{2}{|c|}{ Timing } & \multicolumn{2}{|c|}{ Evolution } \\
\hline & & & Storage & Voiding/postmict & Both & Pre-existing & Post-acute event & Solved & Persisting \\
\hline$\overline{\mathrm{MS}}$ & $13.6 \%$ & 6 & 3 & 2 & 1 & 0 & 6 & 0 & 6 \\
\hline ND & $53.8 \%$ & 7 & 5 & 2 & 0 & 1 & 6 & 3 & 3 \\
\hline PNP & $5.3 \%$ & 0 & 0 & 0 & 0 & 0 & 0 & 0 & 0 \\
\hline ODD & $2.3 \%$ & 0 & 0 & 0 & 0 & 0 & 0 & 0 & 0 \\
\hline NPL & $6.1 \%$ & 0 & 0 & 0 & 0 & 0 & 0 & 0 & 0 \\
\hline PP & $4.5 \%$ & 0 & 0 & 0 & 0 & 0 & 0 & 0 & 0 \\
\hline $\mathrm{SCl}$ & $6.1 \%$ & 1 & 0 & 1 & 0 & 0 & 1 & 0 & 1 \\
\hline Other & $3.8 \%$ & 0 & 0 & 0 & 0 & 0 & 0 & 0 & 0 \\
\hline
\end{tabular}




\section{Discussion}

LUTS are a common problem in both sexes and increases with age. The pathogenesis may be multifactorial and can include LUTD secondary to neurological disorders (6). In the setting of a neurorehabilitation service, it is difficult to estimate the prevalence of LUTS and estimates are varied. Evidence suggests that it can depend on the validated questionnaires used and the different points in time when urinary symptoms are investigated in the neurological pattern disorder (7). However, there are gaps in the literature. Kohler (8) stated, among 126 patients, 78 were afflicted by a disturbance of urinary extraction. Zellner (4) claimed at the time of admission into the rehabilitation centre, $64.9 \%$ of patients presented relevant urological symptoms. In our experience, prevalence of LUTS was only $11 \%$. This new investigation result is not unexpected because we must take into account, in first-order, that this could depend on the different kind of population studied in terms of neurological disorders, age and sex. Second, we must take into account that LUTS following neurological illness may be influenced by multiple factors. For example, the incidence and prevalence of LUTS may rise with increasing progression of the underlying neurological disease (9) but also may vary due to the pattern expected based upon type of neurological lesion (10). Moreover, attention should be paid to concomitant urological conditions, benign prostatic hyperplasia (BPH), idiopathic overactive bladder $(\mathrm{OAB})$, urinary infection; such urological conditions are common in aging patients (11). On the other hand, several factors might influence LUTS as functional and cognitive impairment (grade of severe motor paresis, dysphasia and mental impairment), nocturnal poly-nocturia, diabetes, obesity, stool impaction, etc. (12).

In our experience, LUTS appear to be related to neurological disorders. In general, 93\% of urinary symptoms arise as a result of several neurologic conditions. We believe this high report could be attributed also to other associated factors such as age, sex or grade disability of the population sample examined. In fact many of these outpatients was elderly, females and with critical autonomy .In these cases the presence of neurologic deficits and functional disability more easily decreases the physiological competence of both the bladder function and the sphincter function of the urethra and pelvic floor muscles. It is well know that the process of micturition is controlled by the central nervous system (CNS), which coordinates sympathetic, parasympathetic and somatic nervous system activity for normal micturition and urinary continence. Damage to or diseases of the CNS or within the peripheral or autonomic nervous system may lead to dysfunction in voiding that lead to an inability of the sphincter to appropriately increase or decrease its pressure when bladder pressure is increased (13). A high proportion of these outpatients (64.2\%) presented bothersome urinary symptoms such as incontinence, frequency and urgency (storage LUTS). Many neurogenic patients, especially those with multiple sclerosis, cerebrovascular accidents, and spinal cord injury, experience uninhibited bladder contractions $(14,15)$. The most frequent symptom was urinary urge incontinence (42.8\%). This symptom was identified as prevalent in the multiple sclerosis and neurovascular disorders. The slight prevalence of this kind of uri- nary disorder in the subgroup of patient with cerebrovascular disease, although minor compared to multiple sclerosis, is nevertheless a relevant issue and is probably related with prevalence of neurovascular disorder cases in our neurorehabilitation service. In the literature urge incontinence has been reported in 29\% of stroke patients at 3 month follow up (16) and in 19\% at 6 month follow up (12). Based on Gelber (17) the major mechanism responsible for post stroke urinary incontinence may be the disruption of the neuromicturition pathways, resulting in bladder hyper-reflexia and urge incontinence. A variety of patterns may be seen, but detrusor overactivity of the bladder was identified in 50\% to $90 \%$ of patients with Multiple Sclerosis too (18). Most patients try to inhibit the involuntary bladder contraction by voluntary contraction of the striated sphincter. If this cannot be accomplished, urgency with incontinence may occur. In the period of at least one year of observation, the prevalence of this clinical sample (78.5\%) did not present complete recovery of urological symptoms in spite of the self-reported functional activity limitations were improved. All these patients were referred to the urologist and they are currently in follow up. No one of these patients performed urological rehabilitation. It is a common belief that the medical specialist in neurorehabilitation focuses on only the performance of functional activities and that the urological parameters do not interfere with the outcomes of the rehabilitation process. Current literature reports few data regarding the impact of the urinary symptoms on the outcomes rehabilitation process. Lorenze (3) reported that persistent urinary incontinence was associated with delay or failure of the recovery process with respect to ambulation. Zellner (4) declares that a further optimization of the rehabilitation potential of neurologically ill patients has been regarded as possible by an implementation of urological basic measures into the neurological treatment routine. Likewise the literature data showed significant association between poor wellbeing and LUTS (19); also poor wellbeing includes serious problems of rehabilitation programme. Subjects whose urinary incontinence persisted throughout their rehabilitation programme did not perform significant improvement in rehabilitation. Moreover, they showed to be worsened if we consider Barthel index. Subjects who regained continence had higher weekly Functional Independence Measure (FIM) (20). Therefore, we suggest an increased focus on LUTS in the neurorehabilitation unit and to stress the possibility of a first-line communication between specialist in neurorehabilitation and urologists to implement an earlier, appropriate diagnosis and treatment of LUTS. Finally, the limitation of this study is that, being a retrospective study, it lacks a validated data collection instrument, as the International Prostate Symptom Score (IPSS), but it is based on the analysis of the files of the patients and for this reason urological problems may have been underestimated.

\section{Conclusions}

Neurological disorders are a significant issue in rehabilitation services and they can lead to LUTD and LUTS. Storage symptoms are more common, especially urge incontinence. Not only an adequate neurological assess- 
ment is important for the treatment of these patients, but also an increased attention on urological disorders is advisable to optimise overall outcomes.

A multidisciplinary approach should be advised for the treatment of these patients, involving many different specialists and the whole rehabilitation team.

\section{References}

1. Gulur DM, Drake MJ. Management: of overactive bladder. Nat Rev Urol. 2010; 7:572-582.

2. Li WJ, Oh SJ. Management of lower urinary tract dysfunction in patients with neurological disorders. Korean J Urol. 2012; 53:583592.

3. Lorenze EJ, Simon HB, Linden JL. Urologic problems in rehabilitation of hemiplegic patients. JAMA 1959; 169:1042-1046.

4. Zellner M. Epidemiology of urological symptoms in neurological disorders. A prospective analysis in a center for neurological rehabilitation. Urologe A. 2008; 47:675-84.

5. Abrams P, Cardozo L, Fall M, et al. The standardisation of terminology of lower urinary tract function: report from the standardisation sub-committee of the International Continence Society. Neurourol Urodyn 2002; 21:167-178.

6. Wu MP, Su TH. Do lower urinary tract symptoms (LUTS) predispose the individuals to more admissions in Taiwanese women? - A preliminary report. Taiwan J Obstet Gynecol. 2012; 51:402-4.

7. Brittain KR, Perry SI, Peet SM, et al. Prevalence and impact of urinary symptoms among community-dwelling stroke survivors. Stroke. 2000; 31:886-891.

8. Kohler M, Franer M, Ilko W. Early rehabilitation measures in the area of urologic extraction. Wien Med. Wochenschr. 1991; 141:235-41.

9. Mehnert U, Nehiba M. Neuro/urological dysfunction of the lower urinary tract in CNS diseases :pathophysiology, epidemiology, and treatment options. Urologe A. 2012; 51:189-197.

10. Panicker JN, Menon L, Anandkumar A, et al. Lower urinary tract symptoms following neurological illness may be influenced by multiple factors: observations from a neurorehabilitation service in a developing country. Neurourol Urodyn. 2010: 29:378-81.

11. Wehrberger C, Madersbacher S, Jungwirth S, et al. Lower urinary tract symptoms and urinary incontinence in a geriatric cohort - a population-based analysis. BJU Int. 2012; 110:1516-1521.

12. Nakayama H, Jorgensen HS, Pedersen PM, et al. Prevalence and risk factors of incontinence after Stroke. The Copenhagen Stroke Study. Stroke. 1997; 28:58-62.

13. Ginsberg D. The epidemiology and pathophysiology of neurogenic bladder. Am J Manag Care. 2013; 19 (10 Suppl):191-6.

14.Dorsher PT, McIntosh PM. Neurogenic bladder.Adv Urol 2012; 2012:816274.

15..Linsenmeyer TA, Culkin D. APS recommendations for the urological evaluation of patients with spinal cord injury. J Spinal Cord Med. 1999; 22:139-142.

16.Sakakibara R, Hattori T, Yasuda K, Yamanishi. Micturition disturbance after acute hemispheric stroke: analysis of the lesion site by CT and MRI. J Neurol Sci 1996; 137:47-56.

17. Gelber DA, Good DC, Laven LJ, Verhulst SJ. Causes of urinary incontinence after acut hemispheric stroke. Stroke. 1993; 24:378-382.

18. Manak A, Lurvink M, Barf HA, et al. Epidemiology and healthcare utilization of neurogenic bladder patients in a US clims database. Neurourol Urodyn. 2011; 30:395-401.

19. Tibaek S, Dehlendorff C, Iversen HK, et al. Is well being associated with lower urinary tract symptoms in patient with stroke?.Scand J. Nephrol. 2011; 45:134-42.

20. Gross JC. Urinary incontinence and stroke outcomes. Arch Phys Med Rehabil. 2000; 81:22-7.

\section{Correspondence}

Fabrizio Torelli, MD (Corresponding Author)

fatorelli@tiscali.it

Salvatore Blanco, MD

sblanco_74@yahoo.it

Marco Grasso, MD

m.grasso@hsgerardo.org

Division of Urology, San Gerardo Hospital

via G. Pergolesi, 33 - 20052 Monza (MB)

Erica Terragni, MD

eri_eri66@hotmail.com

Natale Di Bella, MD

nataledibella@libero.it

Donatella Bonaiuti, MD

dbonaiuti2@yahoo.it

Division of Neurorehabilitation, San Gerardo Hospital

via G. Pergolesi, 33 - 20052 Monza (MB) 